\title{
Low-arginine and low-protein diets induce hepatic lipid accumulation through different mechanisms in growing rats
}

\author{
Lila Otani $^{1 \dagger}$, Hiroki Nishi ${ }^{2 \dagger}$, Ayaka Koyama', Yuta Akasaka', Yusuke Taguchi ${ }^{4}$, Yuka Toyoshima ${ }^{4}$, Daisuke Yamanaka ${ }^{3}$, \\ Fumihiko Hakuno ${ }^{2}$, Huijuan Jia', Shin-Ichiro Takahashi ${ }^{2}$ and Hisanori Kato ${ }^{1 *}$ (D)
}

\begin{abstract}
Background: Dietary protein deficiency and amino acid imbalance cause hepatic fat accumulation. We previously demonstrated that only arginine deficiency or total amino acid deficiency in a diet caused significant hepatic triglyceride (TG) accumulation in young Wistar rats. In this study, we explored the mechanisms of fatty liver formation in these models.

Methods: We fed 6-week-old male Wistar rats a control diet (containing an amino acid mixture equivalent to 15\% protein), a low-total-amino acid diet (equivalent to 5\% protein; 5PAA), and a low-arginine diet (only the arginine content is as low as that of the 5PAA diet) for 2 weeks.

Results: Much greater hepatic TG accumulation was observed in the low-arginine group than in the low-totalamino acid group. The lipid consumption rate and fatty acid uptake in the liver did not significantly differ between the groups. In contrast, the low-total-amino acid diet potentiated insulin sensitivity and related signaling in the liver and enhanced de novo lipogenesis. The low-arginine diet also inhibited hepatic very-low-density lipoprotein secretion without affecting hepatic insulin signaling and lipogenesis.

Conclusions: Although the arginine content of the low-arginine diet was as low as that of the low-total-amino acid diet, the two diets caused fatty liver via completely different mechanisms. Enhanced lipogenesis was the primary cause of a low-protein diet-induced fatty liver, whereas lower very-low-density lipoprotein secretion caused low-arginine diet-induced fatty liver.
\end{abstract}

Keywords: Arginine deficiency, Low-protein diet, Hepatosteatosis, Apolipoprotein A-IV, Insulin signaling

\section{Background}

Dietary protein has a strong impact on growth, body composition, and energy homeostasis. Protein overload results in a reduced body fat in humans $[1,2]$ and in a improve insulin sensitivity in rats [3, 4]. By contrast, dietary protein malnutrition induces hepatic fat

\footnotetext{
* Correspondence: akatoq@mail.ecc.u-tokyo.ac.jp

${ }^{+}$Lila Otani and Hiroki Nishi contributed equally to this work.

'Department of Agricultural Biological Chemistry, The University of Tokyo, Bunkyo-ku, Tokyo 113-8657, Japan

Full list of author information is available at the end of the article
}

accumulation, a symptom of kwashiorkor, in rats [5] and humans [6]. The dietary amino acid balance also affects fat deposition in the liver. For example, rats fed lowprotein diets supplemented with essential amino acids, such methionine, experience excessive fat deposition in the liver, and supplementation of a low-protein diet with DL-threonine prevents hepatic fat accumulation [7].

Non-alcoholic fatty liver disease (NAFLD) represents a series of hepatic disorders triggered by excessive liver fat accumulation and is associated with central obesity and insulin resistance. While the effects of dietary fat and

C C The Author(s). 2020 Open Access This article is licensed under a Creative Commons Attribution 4.0 International License, which permits use, sharing, adaptation, distribution and reproduction in any medium or format, as long as you give appropriate credit to the original author(s) and the source, provide a link to the Creative Commons licence, and indicate if changes were made. The images or other third party material in this article are included in the article's Creative Commons licence, unless indicated otherwise in a credit line to the material. If material is not included in the article's Creative Commons licence and your intended use is not permitted by statutory regulation or exceeds the permitted use, you will need to obtain permission directly from the copyright holder. To view a copy of this licence, visit http://creativecommons.org/licenses/by/4.0/ The Creative Commons Public Domain Dedication waiver (http://creativecommons.org/publicdomain/zero/1.0/) applies to the data made available in this article, unless otherwise stated in a credit line to the data. 
carbohydrates on liver fat accumulation have been extensively studied, the effects of dietary protein $[8,9]$ and amino acids [10] on energy homeostasis are less well understood despite their importance in the pathogenesis of NAFLD. For example, insulin is a major anabolic hormone that is intimately involved in hepatic metabolism, and its secretion is affected by dietary protein [11] and amino acid intake [12]. Protein malnutrition suppresses insulin secretion from the pancreas in response to glucose intake $[9,13]$. Some amino acids, such as arginine and leucine, are also known to stimulate insulin secretion $[12,14]$. Moreover, our group previously demonstrated that dietary protein malnutrition decreased plasma insulin and insulin-like growth factor 1 concentrations $[9,15]$, and upregulated the protein expression of insulin receptor substrate 2 (IRS2), one of the key mediators of insulin signaling, in the livers of growing rats, resulting in increased insulin sensitivity [16]. These results implied that enhanced insulin sensitivity caused by dietary protein deficiency may mediate fatty liver development.

We previously investigated the impacts of various amino acids on fat accumulation in the liver using amino acid-deficient diets, and the results showed that the liver triglyceride (TG) content could be increased only by a deficiency of arginine out of the 20 major amino acids tested [10]. Therefore, we hypothesized that lipid accumulation in the liver induced by dietary amino acid deficiency could be attributed to increased insulin sensitivity, which may be caused specifically by dietary arginine deficiency. Here, we tested this hypothesis by evaluating metabolic status and hepatic lipid flux in models of total amino acid and arginine deficiency.

\section{Methods and materials Materials}

Anti-insulin receptor subunit $\beta$ (IR $\beta$ ) and anti-CD36 antibodies for immunoblotting were purchased from Santa Cruz Biotechnology (Dallas, TX, USA). The antiIRS2 (clone 9.5.2) antibody for immunoblotting was purchased from Merck Millipore (Billerica, MA, USA). Anti-phospho-p70 S6K (Thr389), anti-p70 S6K (49D7), anti-phospho-4E-BP1 (Thr37/46), anti-4E-BP1, and antiphospho-AMPKa (Thr172) (40H9) antibodies were purchased from Cell Signaling Technology (Danvers, MA, USA). The anti-GAPDH (6C5) antibody was purchased from Abcam (Cambridge, UK). Anti-rabbit and mouse IgG horseradish peroxidase-conjugated secondary antibodies were purchased from GE Healthcare (Little Chalfont, UK).

\section{Animals}

The experiments were approved by the Animal Usage Committee of the Faculty of Agriculture of the University of Tokyo and performed in accordance with its guidelines (Permission No. P09-375). Male Wistar rats were purchased from Charles River Laboratories International (Kanagawa, Japan). The animals were housed individually in wire cages with free access to food and water. The rats were maintained at a room temperature of $23^{\circ} \mathrm{C} \pm 1{ }^{\circ} \mathrm{C}$ with $50-60 \%$ relative humidity under a 12-h light/dark cycle (light from 08:00 to 20:00). In the pre-experimental period, the rats were fed a purified diet containing $15 \%$ protein from casein.

\section{Comparison of the effects of the low-total-amino acid and low-arginine diets}

Six-week-old male Wistar rats were randomly divided into 3 groups with different diets: the amino acid mixture control diet (equivalent to $15 \%$ protein in the diet; 15PAA), low-arginine diet in which the concentration of arginine in young rodents was $33 \%$ of that in the 15PAA diet (low Arg, $n=8$ ), and the low-total-amino acid diet (equivalent to $5 \%$ protein in the diet; 5PAA). The lowtotal-amino acid mixture simulated the composition of casein [17]. The diet compositions are shown in Tables 1 and 2. Rats were given ad libitum access to tap water and food. Body weight and food intake were measured daily. Fourteen days after initiation of the experimental diets, the rats were anesthetized by intraperitoneal injection of pentobarbital $(30 \mathrm{mg} / \mathrm{kg}) 1 \mathrm{~h}$ after removal of the diet, and a postprandial blood sample was collected from the carotid artery. The liver, longissimus muscles, and abdominal fat were removed and weighed. The livers and muscle tissues were soaked in RNAlater (AMBION, Austin, TX, USA) or snap-frozen in liquid nitrogen and stored at $-80^{\circ} \mathrm{C}$ until use. The organ samples were used to analyze tissue TG concentrations, and protein and RNA expression.

We performed oral glucose tolerance tests (OGTTs) $12 \mathrm{~d}$ after initiation of the experimental diets. After $16 \mathrm{~h}$ of overnight fasting, glucose $(2 \mathrm{~g} / \mathrm{kg})$ was orally administered to the rats. Blood (preprandial) was collected from the tail vein into heparinized tubes that were chilled on

Table 1 Composition of diet

\begin{tabular}{llll}
\hline Component & 15P AA & 5PAA & Low Arg \\
\hline & g/kg diet & & \\
Amino acid mixture $^{\mathrm{a}}$ & 150.0 & 50.0 & 146.5 \\
DL-methionine & 2.5 & 0.8 & 2.5 \\
Corn starch $_{\text {Soy bean oil }}$ & 620.5 & 747.2 & 649.0 \\
Vitamin mixture $^{\mathrm{b}}$ & 50.0 & 50.0 & 50.0 \\
Mineral mixture $^{\mathrm{b}}$ & 10.0 & 10.0 & 10.0 \\
Choline chloride & 40.0 & 40.0 & 40.0 \\
Cellulose powder $^{\text {Composton of }}$ & 2.0 & 2.0 & 2.0 \\
\hline
\end{tabular}

${ }^{a}$ Composition of amino acid mixture were described in Table 2

${ }^{\mathrm{b}} \mathrm{AIN}-76$ prescription (Oriental Yeast Co., Itd) 
Table 2 The amino acid content of each diet

\begin{tabular}{llll}
\hline & 15PAA & 5PAA & Low Arg \\
\hline & g/kg diet & & \\
Alanine & 4.08 & $\mathbf{1 . 3 6}$ & 4.08 \\
Arginine & 5.25 & $\mathbf{1 . 7 5}$ & $\mathbf{1 . 7 5}$ \\
Asparagine- ${ }_{2} \mathrm{O}$ & 5.76 & $\mathbf{1 . 9 2}$ & 5.76 \\
Aspartate & 5.05 & $\mathbf{1 . 6 8}$ & 5.05 \\
Cystine & 0.8 & $\mathbf{0 . 2 7}$ & 0.80 \\
Glutamate & 14.65 & $\mathbf{4 . 8 8}$ & 14.65 \\
Glutamine & 14.65 & $\mathbf{4 . 8 8}$ & 14.65 \\
Glycine & 2.59 & $\mathbf{0 . 8 6}$ & 2.59 \\
Histidine & 4.06 & $\mathbf{1 . 3 5}$ & 4.06 \\
Isoleucine & 7.12 & $\mathbf{2 . 3 7}$ & 7.12 \\
Leucine & 13 & $\mathbf{4 . 3 3}$ & 13.00 \\
Lysine-HCl & 14.1 & $\mathbf{4 . 7 0}$ & 14.10 \\
Methionine & 3.89 & $\mathbf{1 . 3 0}$ & 3.89 \\
Phenylalanine & 7.2 & $\mathbf{2 . 4 0}$ & 7.20 \\
Proline & 14.98 & $\mathbf{4 . 9 9}$ & 14.98 \\
Serine & 8.09 & $\mathbf{2 . 7 0}$ & 8.09 \\
Threonine & 6.09 & $\mathbf{2 . 0 3}$ & 6.09 \\
Tryptophan & 7.76 & $\mathbf{0 . 5 8}$ & 1.73 \\
Tyrosine & 1.73 & $\mathbf{2 . 5 9}$ & 7.76 \\
Valine & 9.16 & $\mathbf{3 . 0 5}$ & 9.16 \\
Total volume & 150.00 & 50.00 & 146.50 \\
\hline & & & \\
& & & \\
& & &
\end{tabular}

ice. The blood samples were subjected to centrifugation at $3000 \times g$ for $5 \mathrm{~min}$ at $4{ }^{\circ} \mathrm{C}$ and the supernatants were transferred to new tubes. Plasma samples were stored at $-80^{\circ} \mathrm{C}$ until analysis.

\section{Blood biochemistry}

Blood biochemical parameters, including total cholesterol, TG, free fatty acids (FFA), and glucose concentrations, were determined using commercial kits (Cholesterol E-test, Triglyceride E-test, NEFA C-test, and Glucose CII test, respectively; Wako Pure Chemical Industries, Osaka, Japan). The plasma insulin concentration was measured using an insulin measurement kit (Morinaga Institute of Biological Science, Yokohama, Japan) according to the manufacturer's instructions.

\section{Lipid extraction and TG measurements}

Lipids were extracted from frozen livers and longissimus muscles via modified Folch method [18] in a 2:1 (vol/ vol) mixture of chloroform/methanol. The extracts were washed with 0.5 volumes of $0.8 \% \mathrm{KCl}$ and centrifuged at $1500 \times g$ for $10 \mathrm{~min}$, and the organic phases were recovered. The TG content in the liver and plasma was also determined using a commercial kit (Wako Pure
Chemical Industries) according to the manufacturer's instructions.

\section{RNA extraction and reverse transcription-polymerase chain reaction}

Total RNA was isolated from homogenized livers using NucleoSpin ${ }^{\circ}$ RNA (Macherey-Nagel, Düren, Germany) according to the manufacturer's instructions. The total RNA concentration was measured with a NanoDrop ${ }^{\circ}$ spectrophotometer (ND-1000, NanoDrop, Wilmington, DE, USA). The quality of the RNA was determined by assessing the A260/ 280 ratio and by agarose gel electrophoresis. The RNA was reverse-transcribed into cDNA using PrimeScript ${ }^{\circ}$ RT Master Mix (Takara Bio, Shiga, Japan). cDNA was amplified using SYBR ${ }^{\circ}$ Premix Ex Taq II (Takara Bio) according to the manufacturer's protocol. We designed the primers for reverse transcription (RT) polymerase chain reaction (PCR) with the design software Primer 3 . $\beta$-actin was used as an endogenous control. The following PCR primers were used: $\beta$-actin $(A c t b)$ forward, 5'-GGAGATTACTGCCCTGGC TCCTA-3', and reverse, 5'-GACTCATCGTACTCCTGC TTGCTG-3'; MTP (Mttp) forward, 5'-AGCAACATGC CTACTTCTTACAC-3', and reverse, 5'-TCACGGGTTC ACTTTCACTG-3'; apolipoprotein A-IV (ApoA4 or Apoa4) forward, $5^{\prime}$-ACCCTCTTCCAGGACAAACTTG-3', and reverse, 5'-CCTTGGTTAGATGTCCACTCAGTTG-3'; and apolipoprotein B (ApoB or Apob) forward, 5'-CCTGTCCA TTCAAAACTACCACA-3', and reverse 5'-CAATGA ACGAATCAGAAGGTGA-3'

\section{Western blotting}

Western blotting analysis was performed as previously described $[9,16]$. In brief, frozen livers were homogenized in homogenizing buffer and centrifuged at 100, $000 \times g$ for $1 \mathrm{~h}$ at $4{ }^{\circ} \mathrm{C}$. The protein content in the supernatant was determined using a Bio-Rad Protein Assay Kit (Bio-Rad, Hercules, CA, USA). Protein extracts were subjected to sodium dodecyl sulfate-polyacrylamide gel electrophoresis and blotted onto polyvinylidene fluoride membranes. The membranes were blocked with blocking buffer, and then incubated at $4{ }^{\circ} \mathrm{C}$ overnight with primary antibodies against IR $\beta$, CD36, and FFA synthase (1: 200 dilution), and against PI3 kinase p85, phospho-p70 S6K (Thr389), p70 S6K, phospho-4E-BP1 (Thr37/46), 4E-BP1, phospho-AMPK $\alpha$ (Thr172), AMPK $\alpha$, phosphoacetyl-CoA carboxylase (Ser79), acetyl CoA carboxylase, and acetyl CoA carboxylase 1 (1:1000 dilution). Primary mouse anti-IRS2 and anti-GAPDH antibodies were used at dilutions of 1:1000 and 1:3000, respectively. We visualized the blots by chemiluminescence after incubating with donkey anti-rabbit IgG or sheep anti-mouse IgG conjugated to horseradish peroxidase (1:2500). The immunoreactive bands were exposed and the signals were 
quantified using a cooled charge-coupled device camera system (LAS-3000 Mini; Fujifilm, Kanagawa, Japan).

\section{Very-low-density lipoprotein excretion test}

Male Wistar rats were fed a casein control diet between 10:00 and 18:00 for $7 \mathrm{~d}$ prior to the experiment. After habituation, the rats (7.5 weeks of age, 208-229 g) were assigned to the 15PAA $(n=8)$, 5PAA $(n=8)$, and low Arg $(n=9)$ groups. The diet compositions are shown in Tables 1 and 2. The experimental diets were provided for $5 \mathrm{~h}$ from 9:00 to 14:00. One hour after removing the diets, we administered tyloxapol $(200 \mathrm{mg} / \mathrm{kg}$, dissolved in $0.9 \% \mathrm{NaCl}$; Triton WR-1339, Sigma-Aldrich, St. Louis, $\mathrm{MO}, \mathrm{USA})$ to all rats under isoflurane anesthesia (3-4\%, 5 l/min; Dainippon Sumitomo Pharma, Osaka, Japan) via the tail vein. Blood was collected from the tail vein into chilled, heparinized tubes prior to tyloxapol injection and $30,60,120$, and $240 \mathrm{~min}$ after injection to measure plasma TG concentrations. Tyloxapol inhibits endogenous lipoprotein lipase and blocks the clearance of lipidcarrying lipoproteins in the blood [19-21]. Thus, the rise in plasma TG concentration after tyloxapol injection is approximately proportional to the very-low-density lipoprotein (VLDL)-TG level. The TG concentrations in the plasma were measured with a commercial kit as described in "Lipid extraction and TG measurements." The TG secretion rate was expressed in $\mathrm{mg} \mathrm{TG} / \mathrm{dl} / \mathrm{min}$.

\section{Respiratory exchange ratio}

Six-week-old male Wistar rats were assigned to the 15PAA $(n=6)$, 5PAA $(n=6)$, and low $\operatorname{Arg}(n=6)$ groups. Six days after initiation of the experimental diets, animals were individually placed in a metabolic chamber for $24 \mathrm{~h}$, and $\mathrm{VO}_{2}$ and $\mathrm{VCO}_{2}$ were monitored every 10 min with an OXYMAX system (Columbus Instruments, Columbus, OH, USA). Rats were allowed free access to water and food during the experiment.

Theoretically, a respiratory exchange rate (RER) of 1.0 represents the dominant consumption of carbohydrates, and a decrease in the RER to approximately 0.7 represents proportionally higher lipid consumption.

\section{De novo lipogenesis assay}

De novo lipogenesis was measured based on the method described previously with minor modifications [22]. Sixweek-old male Wistar rats were assigned to the 15PAA $(n=8)$, 5PAA $(n=8)$, and low Arg $(n=8)$ groups (240$280 \mathrm{~g}$ ) for 1 night (from 18:00 to 10:00). Rats were allowed free access to food. The following day, the rats of each group were further divided into $\mathrm{D}_{2} \mathrm{O}$ (Sigma Aldrich, St. Louis, MO, USA) or $\mathrm{H}_{2} \mathrm{O}$ injection groups (5 $\mathrm{ml} / \mathrm{kg}$ body weight, intraperitoneally). Liver samples were collected under isoflurane anesthesia $(3-4 \%, 5 \mathrm{l} /$ min; Dainippon Sumitomo Pharma, Osaka, Japan) $24 \mathrm{~h}$ after injection and stored at $-80^{\circ} \mathrm{C}$ until analysis. Lipids were extracted as described in "Lipid extraction and TG measurements" [18]. The extracted lipids were hydrolyzed and methylated with an FFA Methylation Kit (Nacalai Tesque, Kyoto, Japan), and the obtained products were purified with a Fatty Acid Methyl Ester Purification Kit (Nacalai Tesque). Purified FFA esters were analyzed by gas chromatography-mass spectrometry (GCMS-QP2010 Plus, SHIMADZU, Kyoto, Japan) to quantify palmitate isotopomers. The relative amounts of fatty acids were normalized by tissue weight and the difference in the total amount of methyl palmitate isotopomers in the $\mathrm{D}_{2} \mathrm{O}$-injected group and $\mathrm{H}_{2} \mathrm{O}$-injected group was interpreted as the de novo lipogenesis rate.

\section{Hepatic FFA uptake assay}

We performed an in vivo FFA uptake assay according to the method published in previous reports [23, 24]. Sixweek-old male Wistar rats were assigned to the 15PAA $(n=8)$, 5PAA $(n=8)$, and low Arg $(n=8)$ groups (240$280 \mathrm{~g}$ ) for 1 night (from 18:00 to 10:00). The following day, the rats of each group were further divided into 2 groups; one was treated with fluorescent FFA analog $\left(0.1 \mathrm{mg} / \mathrm{kg}\right.$, BODIPY $^{\odot} \mathrm{FL} \mathrm{C}_{12}$, Invitrogen, Carlsbad, CA, USA) and the other was treated with only vehicle $(0.1 \%$ bovine serum albumin in phosphate-buffered saline) by intravenous injection under isoflurane anesthesia (3-4\%, 51/min; Dainippon Sumitomo Pharma, Osaka, Japan). The liver samples were collected 10 min after injection and stored at $-80^{\circ} \mathrm{C}$ until analysis. Lipids were extracted [18] and reconstituted in isopropanol. Then, lipid fluorescence was measured with an ARVO X3 microplate reader (PerkinElmer, Waltham, MA, USA). All values were normalized by tissue weight, and the value of the vehicle-administered group was subtracted as background fluorescence from that of the BODIPYadministered group.

\section{Statistical analysis}

All data are presented as the mean \pm standard error. The data were statistically analyzed with the Ekuseru-Toukei 2010 software package (Social Survey Research Information, Tokyo, Japan). Statistical significance was calculated using one-way analysis of variance (ANOVA) with the Bonferroni and Tukey-Kramer post-hoc tests to assess differences between groups. $P<0.05$ was considered statistically significant.

\section{Results}

Diet-induced changes in the body composition of rats Body weight gain was slightly lower in the 5PAA group than in the 15PAA group, whereas the weight gain of the low Arg group was comparable to that of the 15PAA group (Table 3). Liver TG levels were significantly 
Table 3 Blood biochemical parameters in the postprandial condition after feeding the low-protein and low-arginine diets for 14 days

\begin{tabular}{llll}
\hline & 15PAA & 5PAA & Low Arg \\
\hline $\mathrm{n}$ & 8 & 8 & 9 \\
Body weight $(\mathrm{g})$ & $286+/-5^{\mathrm{a}}$ & $267+/-5^{\mathrm{b}}$ & $283+/-5^{\mathrm{a} \mathrm{b}}$ \\
Total cholesterol $(\mathrm{mg} / \mathrm{dl})$ & $69.9+/-2.8^{\mathrm{a}}$ & $65.0+/-2.0^{\mathrm{a}}$ & $50.8+/-2.3^{\mathrm{b}}$ \\
HDL cholesterol $(\mathrm{mg} / \mathrm{dl})$ & $14.8+/-0.6$ & $11.6+/-0.7$ & $13.7+/-0.5$ \\
Glucose $(\mathrm{mg} / \mathrm{dl})$ & $202+/-8$ & $196+/-9$ & $209+/-9$ \\
Insulin $(\mathrm{ng} / \mathrm{ml})$ & $3.2+/-0.2^{\mathrm{ab}}$ & $1.9+/-0.4^{\mathrm{a}}$ & $4.6+/-0.6^{\mathrm{b}}$ \\
\hline
\end{tabular}

Data are shown as the mean $+/-$ S.E.M.

Different superscript letters denote significant differences $(p<0.05)$

higher in the 5PAA group than in the 15PAA group (Fig. 1b, $p<0.01$ ) as previously reported $[9,16]$; however, even higher TG levels were observed in the low Arg group than in the 5PAA group (Fig. $1 \mathrm{~b}, p<0.01$ vs. 15PAA and 5PAA). Macroscopic observations also supported this result; the livers of the 5PAA and low Arg groups were lighter in color than those of the 15PAA group (Fig. 1a). The TG content in the longissimus muscle and the visceral fat volume were significantly higher in the 5PAA group compared to the 15PAA group, whereas these parameters were not affected by the low Arg diet (Fig. 1c, e). Liver weight was significantly higher in the low Arg group than the 15PAA group, but this was not the case for the 5PAA group (Fig. 1d). These data suggested that, in contrast to our expectation, a low-total-amino acid diet and lowarginine diet might lead to different phenotypes, given that the low Arg diet did not completely recapitulate the effects of 5PAA administration.

\section{Diet-induced changes in blood biochemical parameters}

The blood glucose and insulin levels were over $190 \mathrm{mg} /$ $\mathrm{dl}$ and $1.9 \mathrm{ng} / \mathrm{ml}$, respectively, in the postprandial blood (Table 3, Supplementary Table 1). No differences were observed in the FFA concentrations of the fasting and postprandial blood among the 3 groups (Fig. $2 \mathrm{a}$ and $\mathrm{b}$ ). By contrast, postprandial plasma TG and total cholesterol levels were significantly lower in the low Arg group than in the 15PAA group (Fig. 2d, Table 3), whereas the 5PAA diet did not affect plasma TG levels of the preprandial and postprandial blood (Fig. 2c and d). The high-density lipoprotein cholesterol concentrations were similar among the 3 groups in the preprandial and postprandial blood (Table 3).

\section{Glucose tolerance}

When the OGTT was performed at $12 \mathrm{~d}$ after initiation of the experimental diets, the plasma glucose levels of the 5PAA group were lower than those of the 15PAA group at 30 and $60 \mathrm{~min}$ after glucose loading (Fig. 3a). In addition, the plasma insulin levels were significantly lower in the 5PAA group than in the 15PAA group at $30 \mathrm{~min}$ after glucose loading (Fig. 3b). The fasting plasma glucose levels in the low Arg group were higher than in the 15PAA group (Fig. 3a), whereas the fasting plasma insulin levels were not significantly different between the low Arg and 15PAA groups (Fig. 3b). The plasma glucose level after glucose loading was not affected by the low Arg diet. The plasma insulin levels increased less in the low Arg group after glucose loading than in the 5PAA and 15PAA groups. The plasma insulin levels fell at $60 \mathrm{~min}$ after glucose loading in the 15PAA group but did not decrease in the low Arg group.

\section{Hepatic insulin receptor signaling}

Western blotting analysis revealed that the expression of IR $\beta$ was slightly, but not significantly, higher in the livers of 5PAA-fed rats than those of the 15PAA-fed rats (Fig. 4a and b). Rats fed the 5PAA diet exhibited markedly higher expression levels of IRS2 (Fig. 4a and c) in the liver than rats fed the 15PAA or low Arg diet. Expression of the downstream effector 4E-BP1 (Fig. 4a and d) and its threonine phosphorylation were significantly higher in the 5PAA group than in the 15PAA or low Arg group (Fig. 4a and e). In contrast, low Arg-fed rats showed a lower total IR $\beta$ level than the 15PAA- and 5PAA-fed rats, and IRS2, 4E-BP1, and phosphorylated 4E-BP1 levels were comparable to those of 15PAA-fed rats. These results further indicated that the 5PAA and low Arg diets had disparate effects on liver insulin signaling.

\section{TG secretion, de novo lipogenesis, FFA uptake, and energy consumption}

We next assessed hepatic lipid flux in rats fed the 5PAA and low Arg diets. For the hepatic TG secretion assay, the rats were fed the indicated experimental diets for $5 \mathrm{~h}$ after a 15-h fast, and then tyloxapol was injected into the tail vein. The rats in the 3 groups consumed approximately equal amounts of the diets during the $5 \mathrm{~h}$ of feeding (15PAA: $13 \pm 1 \mathrm{~g}$; 5PAA: $14 \pm 1 \mathrm{~g}$; and low Arg: $14 \pm 0 \mathrm{~g})$. When plasma TG concentrations were measured at $0,30,60,120$, and 240 min after tyloxapol injection, every group showed time-dependent increases, but the rate of increase of the low Arg group was significantly lower than that of the 15PAA group (Fig. 5a). Remarkably, the hepatic TG secretion rate of the 5PAA group did not significantly change compared to that of the 15PAA group (Fig. 5b). Analysis of hepatic FFA uptake activity using a fluorescent FFA analog showed comparable levels among the 3 groups, although the expression of CD36 in the liver, which mediates cellular FFA uptake, was significantly lower in the low Arg group 
A

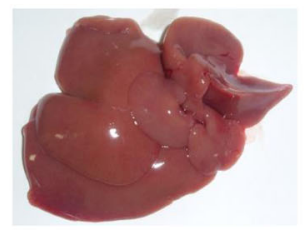

B

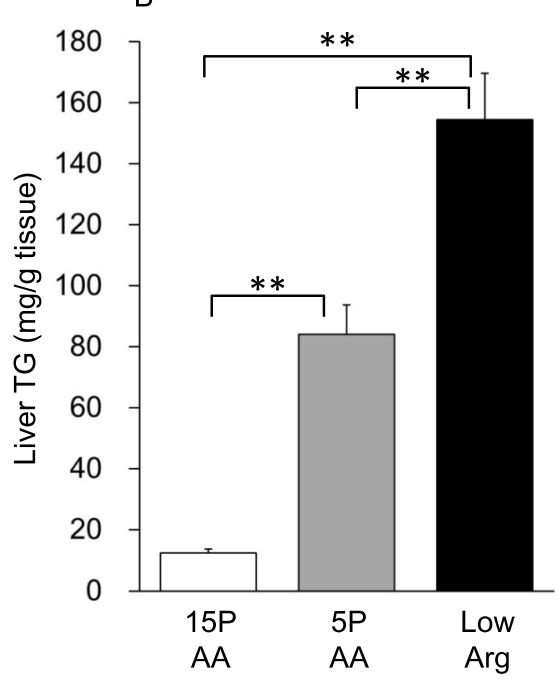

D

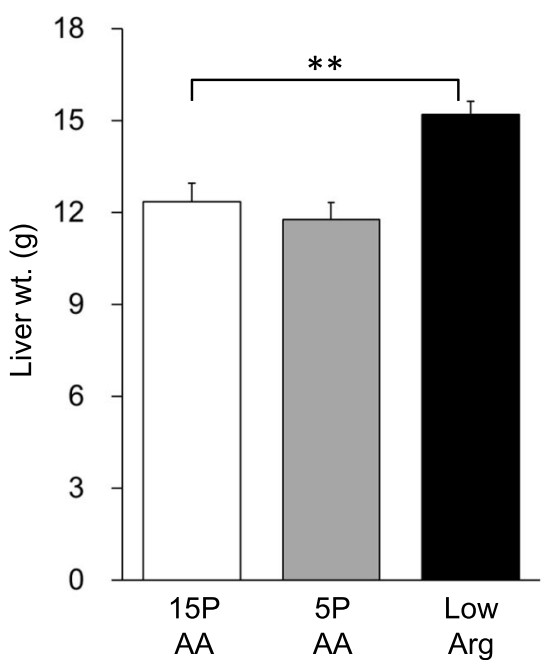

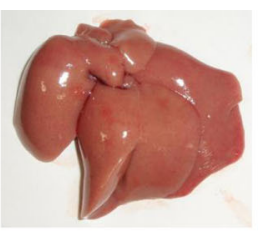

5PAA

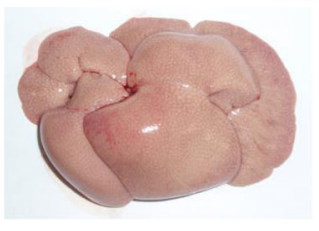

Low Arg

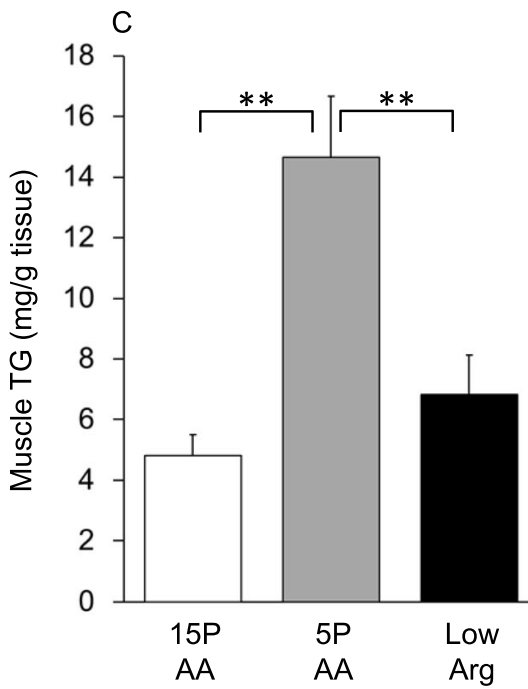

$\mathrm{E}$

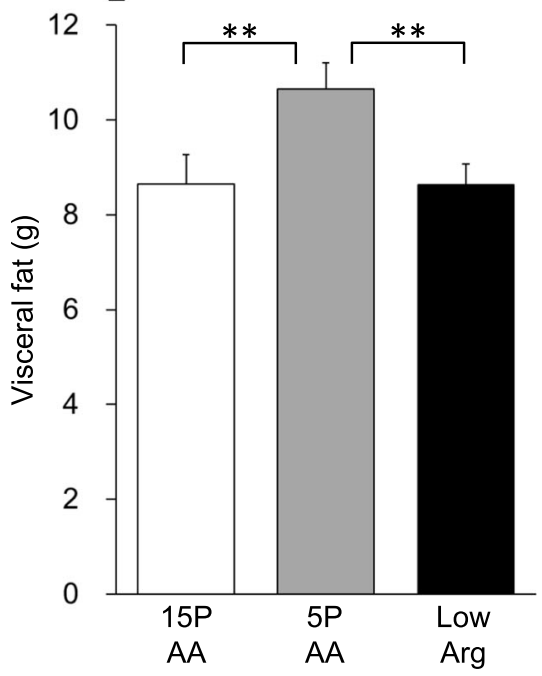

Fig. 1 Effects of the 5PAA and low Arg diets on the liver. Macroscopic liver characteristics (a), hepatic (b) and longissimus muscle (c) TG contents, liver weight (d), and visceral fat weight (e) in 6-week-old male Wistar rats fed the 15PAA $(n=8)$, low $\operatorname{Arg}(n=8)$, and 5PAA ( $n=8)$ diets for 14 d. The values are means \pm standard errors. Asterisks indicate significant differences as assessed by one-way ANOVA with Bonferroni and TukeyKramer post-hoc tests $\left({ }^{*} p<0.05 ;{ }^{* *} p<0.01\right)$

than in the other groups (Fig. 4a and f, c). In addition, the de novo lipogenesis assay demonstrated that the lipid synthetic activity in the liver was enhanced only in the 5PAA group, whereas it was unchanged in the low Arg group (Fig. 5d).

We also measured the RER of the 3 groups (Fig. 5e, Supplementary Figure 2). RER is a stoichiometric ratio of exhaled $\mathrm{CO}_{2}$ to inhaled $\mathrm{O}_{2}$, which roughly reflects the oxidized energy source. The RERs of the 3 groups measured during the night were almost comparable at around 1.0, whereas the RERs measured during the day were slightly lower in the 5PAA and low Arg groups than in the 15PAA group, indicating that the 5PAA diet and low Arg diet did not slow lipid consumption in comparison with the 15PAA diet. 

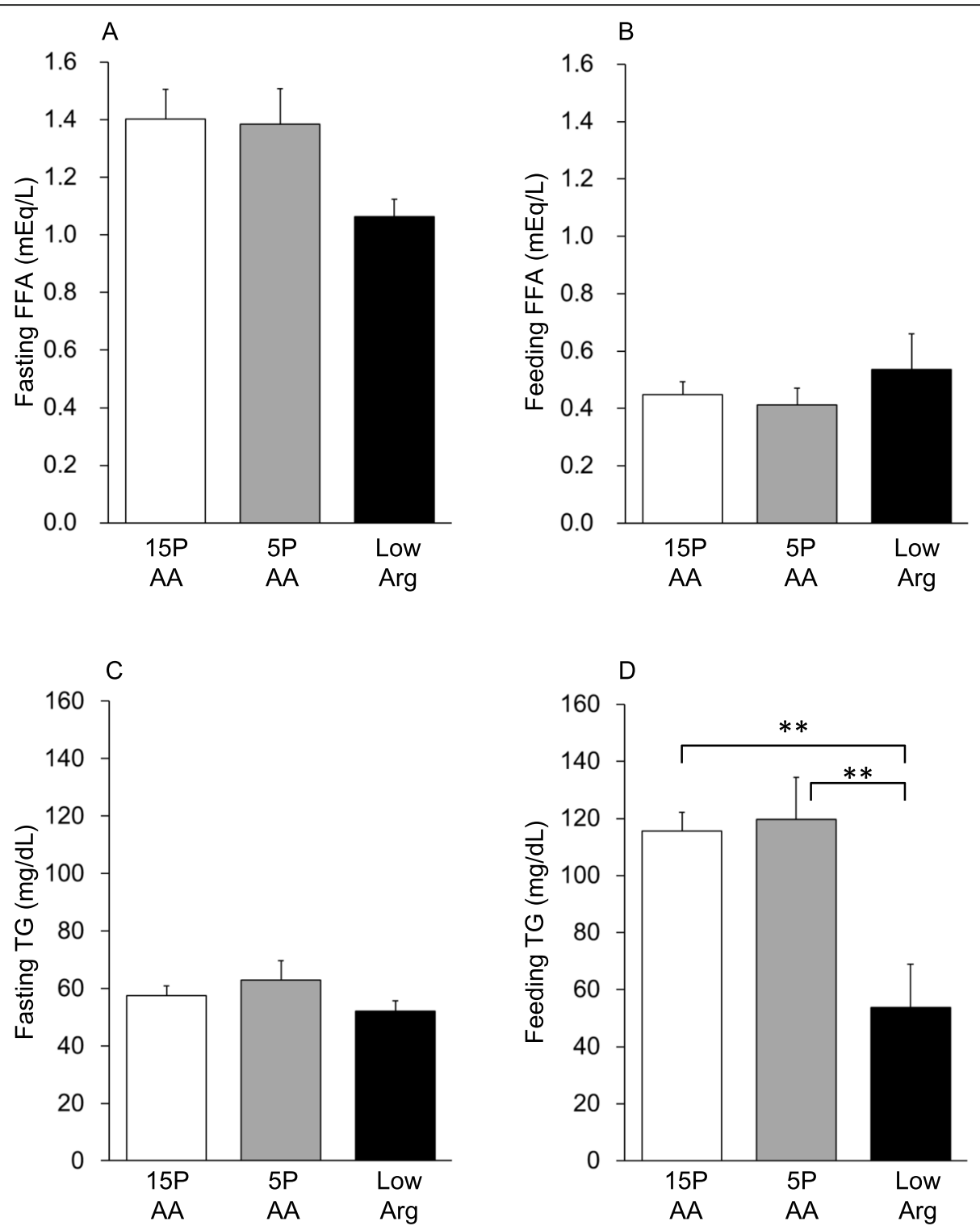

Fig. 2 Plasma free fatty acid and TG levels in preprandial or postprandial conditions after feeding the 5PAA and low Arg diets. Six-week-old male Wistar rats were fed the 15PAA $(n=8)$, low Arg $(n=8)$, and 5PAA $(n=8)$ diet for 14 d. Preprandial free fatty acid $(\mathbf{a})$ and TG (c) levels and postprandial free fatty acid (b) and TG (d) levels are shown. The values are the means \pm standard errors. Asterisks indicate significant differences as assessed by one-way ANOVA with Bonferroni and Tukey-Kramer post hoc tests ( ${ }^{* *} p<0.01$ )

Hepatic TG secretion-related gene expression in the liver We performed microarray analysis of liver samples from the rats of the 3 groups to evaluate the effects of the diets on hepatic transcripts (Supplementary Table 2). The results showed a marked decrease $(0.47$-fold) in the expression of the ApoA4 gene in the livers of the low Arg group compared with that of the 15PAA livers, which was validated by quantitative PCR analysis. ApoA4, an apolipoprotein primarily expressed in the intestine in mammals, is believed to be involved in chylomicron formation [25]. However, in rodents, it is reportedly also expressed in the liver where it is associated with VLDL formation and TG secretion [20].

There were no significant differences in $A p o B$ and $M t t p$ mRNA levels, which are also important genes for hepatic VLDL formation, between the 15PAA and low Arg groups (Fig. 6a, b), whereas there was a tendency of Mttp mRNA levels to be slightly increased in the livers of the rats in the 5PAA group (Fig. 6A, $p<0.08$ vs. 15PAA). However, the low Arg diet caused a dramatic decrease in ApoA4 mRNA levels compared with the 15PAA diet, whereas the 5PAA diet had no effect (Fig. 6c). 

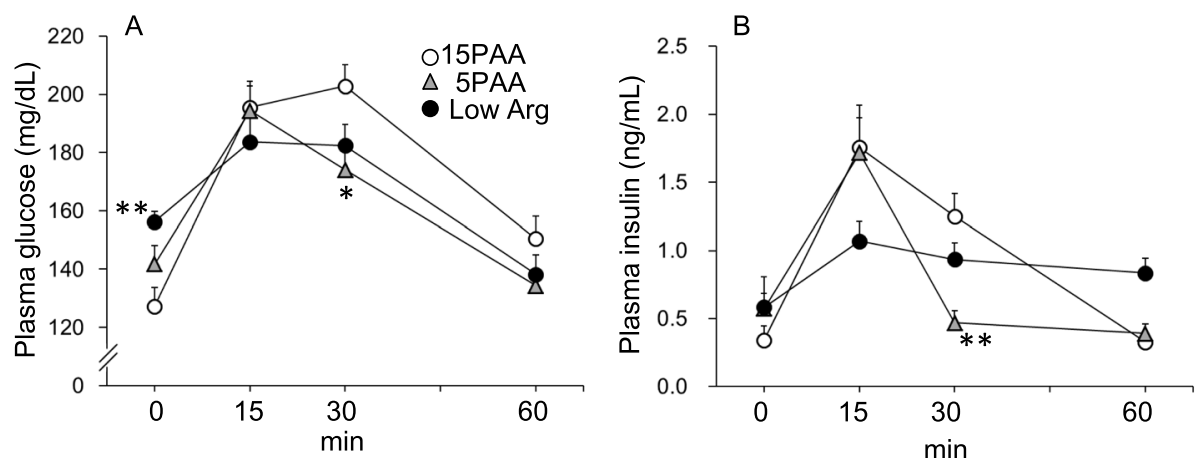

Fig. 3 Effects of the 5PAA and low Arg diets on blood glucose and insulin levels after glucose loading. Six-week-old male Wistar rats were fed the 15PAA $(n=8)$, low Arg $(n=8)$, and 5PAA $(n=8)$ diets, and then OGTTs were performed on d 12 after initiation of the experimental diets. Plasma glucose (a) and insulin (b) concentrations are shown. The values are the means \pm standard errors. Asterisks indicate significant differences as assessed by one-way ANOVA with Bonferroni and Tukey-Kramer post-hoc tests $\left({ }^{*} p<0.05 ;{ }^{* *} p<0.01\right)$

\section{Discussion}

We previously reported that both dietary total amino acid deficiency and selective arginine deficiency caused fatty liver, and activation of insulin receptor signaling is known to stimulate lipid accumulation $[9,16]$. Therefore, we speculated that the phenotypes caused by a low Arg diet simply reflected those caused by the 5PAA diet, which could be mediated through enhanced insulin sensitivity. However, in the present study, we identified several distinct phenotypes in the 5PAA and low Arg groups, including the lipid accumulation level, serum lipid profile, glucose tolerance, and insulin signal activities. In addition, because liver TG content is thought to be determined by the integration of de novo lipogenesis, TG secretion, FFA uptake, and lipid oxidation [26], we evaluated these activities in rats fed the 5PAA or low Arg diet. Contrary to our expectations, these parameters also completely differed between the groups; only the 5PAA group exhibited increased lipogenesis, only the low Arg group showed attenuated TG secretion, and FFA uptake and RER scarcely differed among the groups. The RER was slightly lower, particularly during the day, in the 5PAA and low Arg groups than in the 15PAA group, which indicated that the lipid oxidation rates of the experimental groups were almost the same or slightly higher than that of the 15PAA group. However, as the RER indicates the opposite of lipid accumulation, it cannot be responsible for fatty liver development. Collectively, these results indicate that distinct mechanisms underlie the fatty liver formation induced by total amino acid deficiency and only arginine deficiency.

IR $\beta$ and IRS 2 are pivotal mediators of insulin activity in the liver. We found that the 5PAA diet significantly increased the protein expression levels of total IRS2 and 4EBP1 and 4EBP1 phosphorylation ( $p<0.05$ in comparison with 15PAA), and tended to increase total IR $\beta$ expression in the liver. This suggests that the 5PAA diet may potentiate insulin signaling. The activation of this pathway is known to upregulate FFA synthesis [9, 27-29], and, indeed, increased de novo lipogenesis was observed in 5PAA diet-fed rats. In contrast, we recently reported that when hepatocytes were cultured in an amino acidsufficient or -deficient medium, the intracellular TG level was increased by amino acid deficiency without the addition of any lipids or hormones, and was accompanied by enhanced lipid synthesis [10]. These results indicate that hepatocytes themselves monitor extracellular amino acid concentrations to induce lipid accumulation in a cellautonomous manner. It is possible that both the lipogenesis enhanced by insulin signal potentiation and that stimulated directly by amino acid deficiency may lead to lipid accumulation in the liver in response to 5PAA feeding. On the other hand, the low Arg diet decreased IR $\beta$ and did not have any statistically significant effects on IRS2 and 4E-BP1 protein levels. These findings support the conclusion that the 5PAA and low Arg diets induced hepatic fat accumulation via different mechanisms. 5PAA diet-induced TG accumulation appears to be mediated through insulin signaling, whereas low Arg diet-induced accumulation was insulin-independent.

We also demonstrated that only the 5PAA diet induced visceral and muscular fat accumulation, in addition to accumulation in the liver. Some reports have shown that a low-protein diet leads to intramuscular and other fat deposition in piglets [30-32], and a highprotein diet reduces visceral fat and hepatic TG levels in humans [2, 33]. In addition, we previously found that the activation of insulin signaling in response to consumption of the 5PAA diet was specific to the liver and was not observed in the white adipose tissue or muscle [9]. Taken together, these results suggest that the dietary protein content and amino acid balance can provoke a tissue-specific reaction, and are, thus, important determinants of body fat deposition in mammals. 

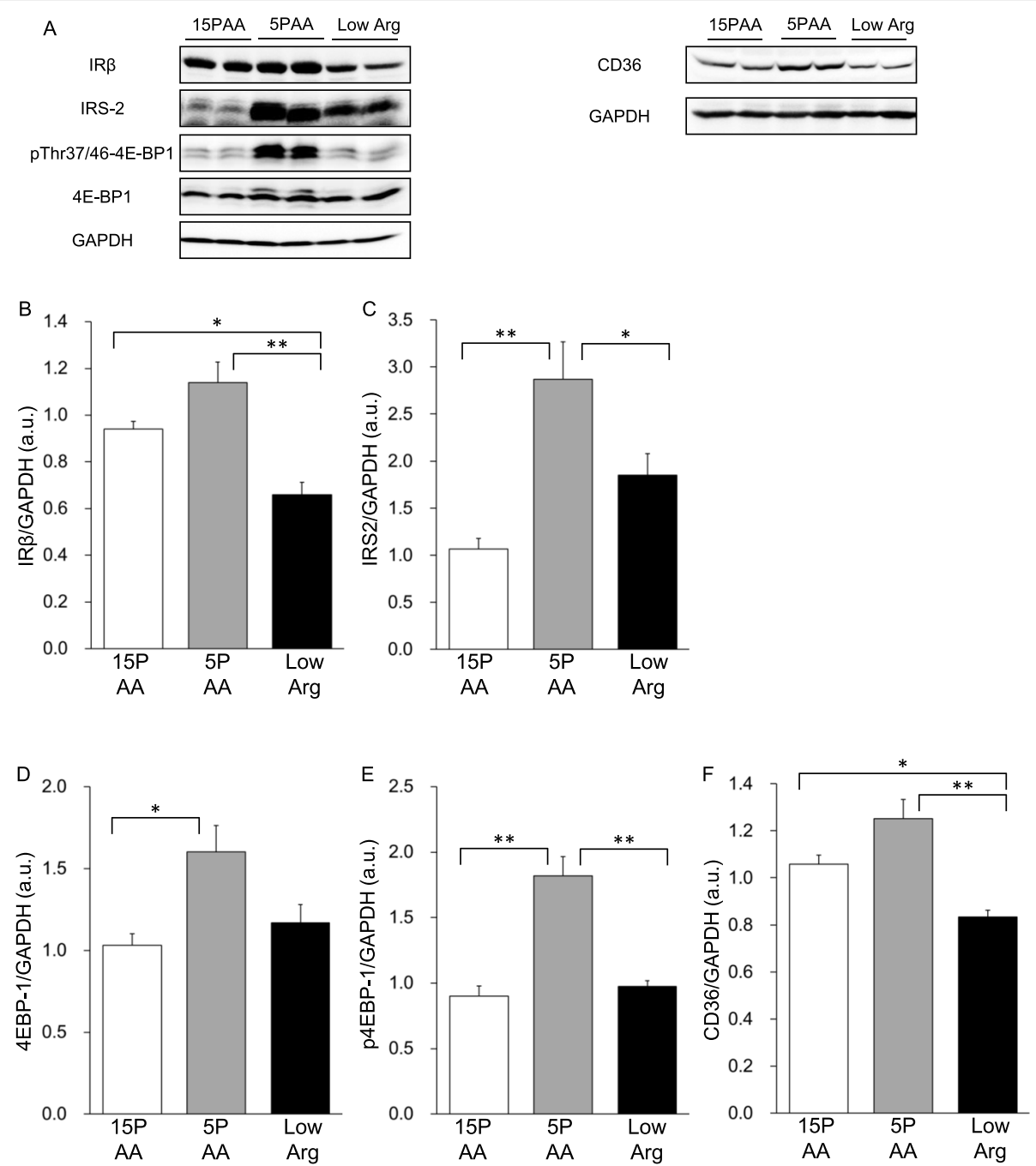

Fig. 4 Insulin signaling-related protein levels in rats fed diets low in protein or arginine. Six-week-old male Wistar rats were fed the 15PAA $(n=8)$, low Arg $(n=$ 8), and 5PAA $(n=8)$ diets for $14 \mathrm{~d}$. Whole-liver lysates were analyzed by immunoprecipitation and immunoblotting with antibodies against IR $(\mathbf{b})$, IRS-2 (c), 4EBP1 (d), phosphorylated 4E-BP1 (Thr37/46) (E), CD36 (f), and GAPDH as an internal control. Representative immunoblots (a) are shown. The values are the means \pm standard errors. Asterisks indicate significant differences as assessed by one-way ANOVA with Bonferroni and Tukey-Kramer post-hoc tests $\left({ }^{*} p<0.05 ;{ }^{* *} p<0.01\right)$

The assembly and secretion of VLDL are the key regulatory steps of plasma and intrahepatic TG and FFA levels owing to its glyceride-rich structure, and are closely linked to the progression of NAFLD. The 5PAA diet had no effect on postprandial plasma TG and fasting FFA levels, whereas the low Arg group had lower postprandial plasma TG and liver CD36 levels than the control group, although their fasting FFA levels were comparable. By contrast, neither the 5PAA nor low Arg diet influenced FFA uptake by the liver. The scavenger FFA receptor CD36 is known to mediate FFA uptake into the metabolic tissues, and is reportedly associated with non-alcoholic fatty liver development [23, 34]. However, in our model, changes in the hepatic CD36 level and blood TG/FFA levels did not affect FFA uptake into the liver, suggesting that the change in serum lipid concentrations was unlikely to be a direct reason for fatty liver formation.

In this study, we analyzed the VLDL secretion rate after tyloxapol injection. Tyloxapol is a non-ionic detergent that prevents the catabolism of triacylglycerol-rich lipoproteins by lipoprotein lipase [19, 35]; it is widely used for the in vivo determination of the secretion and clearance rates of VLDL [21]. We found that VLDL secretion in the 5PAA group was not significantly different to that in the 15PAA group, whereas that of the low Arg group was significantly lower. These findings suggested that the hepatic lipid accumulation caused by the low Arg diet, but not the 5PAA diet, was attributable to the downregulation of VLDL secretion. As the inhibition of 


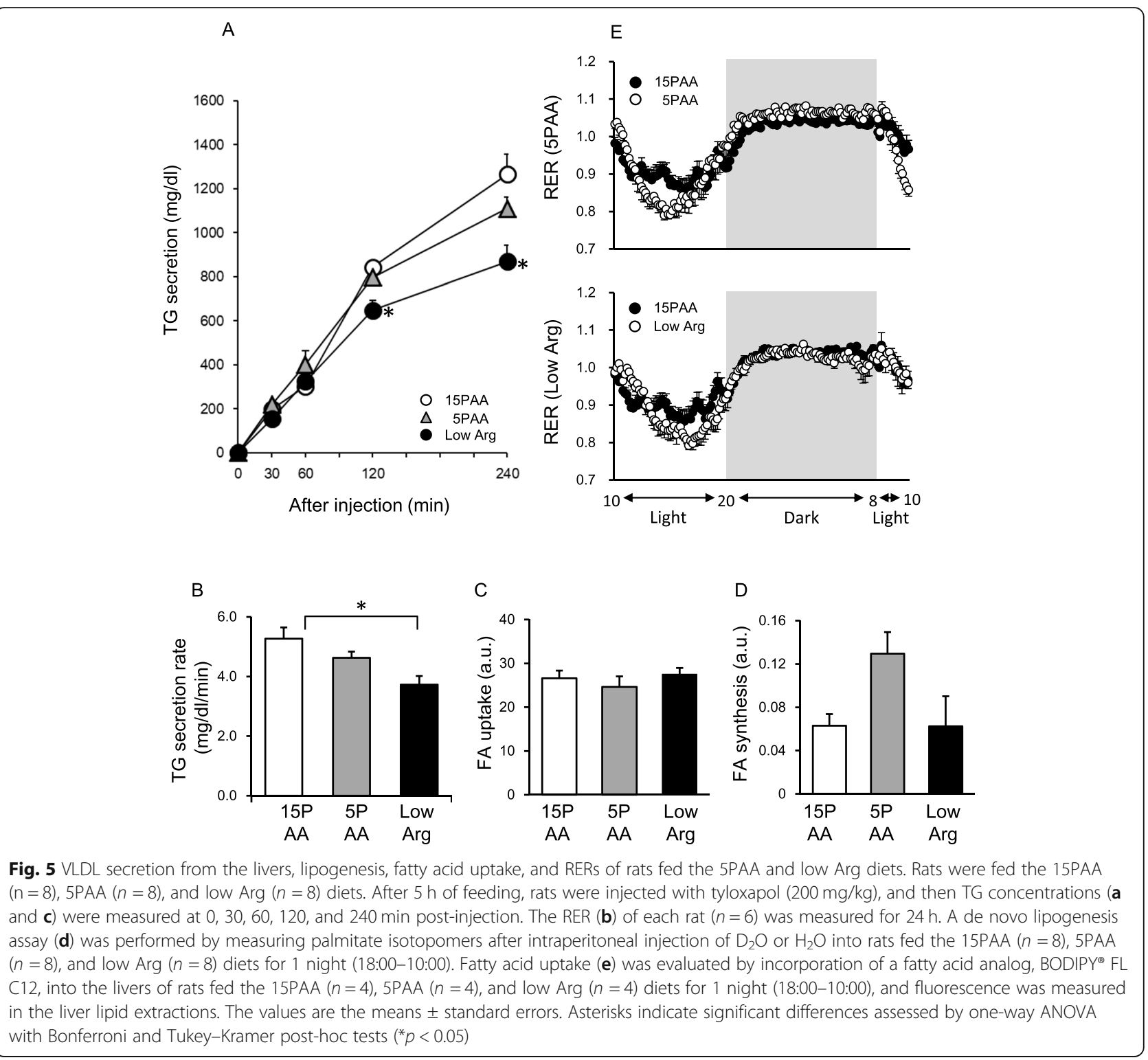

VLDL secretion was observed within just $5 \mathrm{~h}$ of the initiation of the low Arg diet, it may be the major cause of fatty liver formation induced by the low Arg diet.

Although Mttp and ApoB are the most important elements in VLDL assembly [36], neither the 5PAA nor the low Arg diet statistically affected the expression of these genes. Instead, we discovered a decrease in the expression level of ApoA4. ApoA4, a 46-kDa protein with a lipid-binding site, is expressed in the intestine and liver of rodents [25]. It is generally believed to be expressed primarily in the small intestine in humans, although minor expression in the human liver and human hepatocyte lines has also been reported [37, 38]. Adenoviral overexpression of ApoA4 in the liver increases VLDL secretion and decreases hepatic TG levels, and ApoA4knockout mice show lower VLDL secretion than wild- type controls [20]. Thus, ApoA4 is another important apolipoprotein for VLDL formation. Based on these findings, we believe that the downregulation of ApoA4 expression in response to the low Arg diet may attenuate VLDL secretion.

Arginine and lysine use the same membrane transporter system. Lysine is an important amino acid for growth and nutritional metabolism. It tends to be the first limiting amino acid in a variety of circumstances. Low dietary lysine in piglets drives intramuscular fat accumulation and a reduction in serum glucose levels in growing pigs [39]. In our previous study, however, feeding rats a low Arg diet did not affect serum arginine and lysine concentrations, which was presumably due to the compensatory synthesis of arginine. On the other hand, the serum levels of other amino acids, such as 

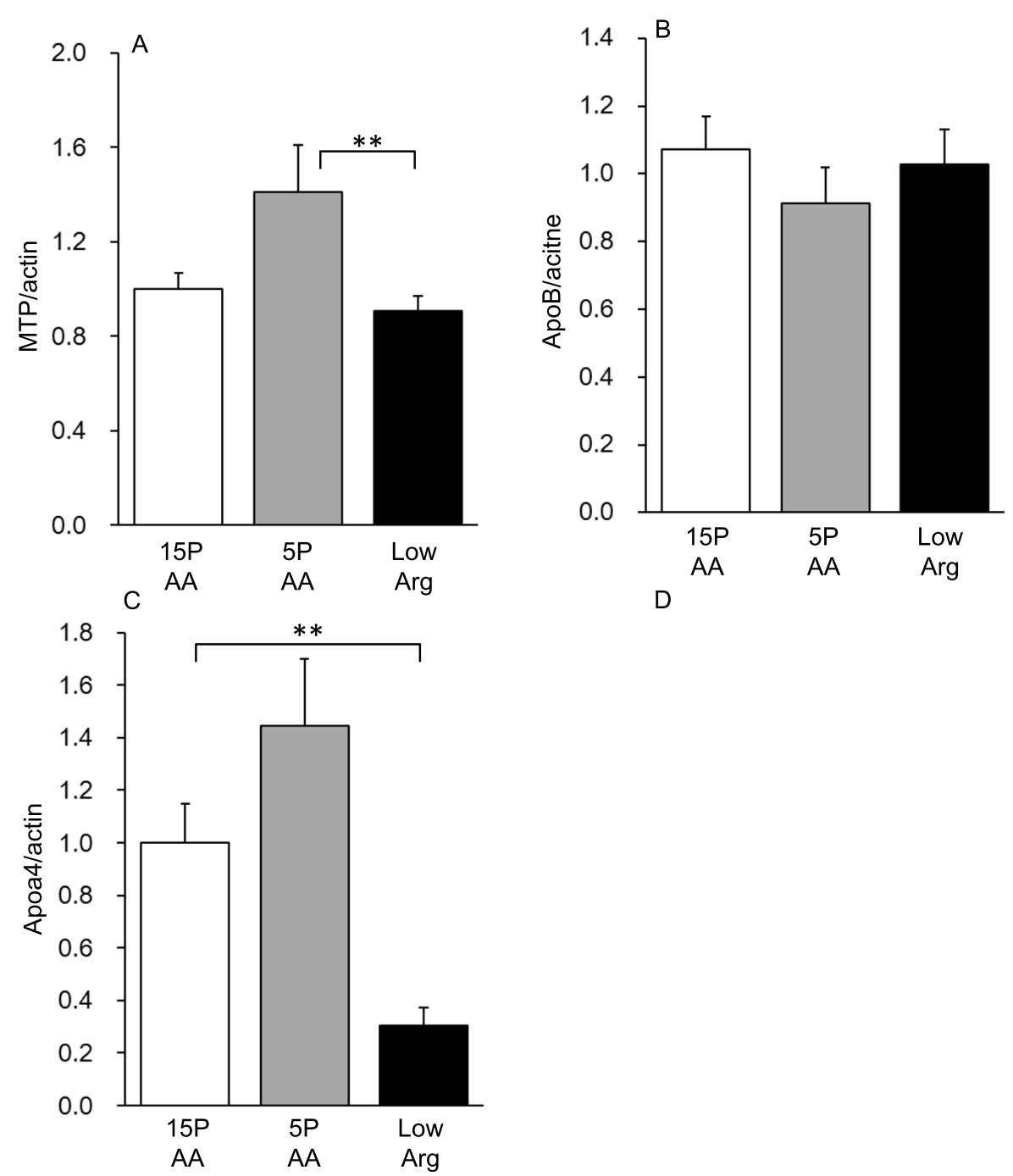

Fig. 6 Effects of the 5PAA and low Arg diets on the expression of VLDL assembly-related genes. Six-week-old male Wistar rats were fed the 15PAA $(n=8)$, low Arg $(n=8)$, and 5PAA $(n=8)$ diets for $14 \mathrm{~d}$. Total RNA was isolated from the liver and then subjected to quantitative RT-PCR. The transcript expression levels for Mttp (a), $A p o B(\mathbf{b})$, and $A p o A 4$ (c) are shown. Asterisks indicate significant differences as assessed by one-way ANOVA with Bonferroni and Tukey-Kramer post-hoc tests $\left({ }^{* *} p<0.01\right)$

methionine and histidine, were significantly increased by a low Arg diet [10]. Therefore we do not consider arginine alone a signal molecule. Instead, we believe that the complex alteration of the comprehensive serum amino acid profile is important. In support of that hypothesis, in the same previous report, a nonlinear analysis based on a mathematical machine learning method revealed that the comprehensive amino acid profile correlated with TG levels in the liver although no single amino acid concentration correlated well. The molecular basis of how such information is sensed by cells remains unclear. We are currently investigating the molecular sensing mechanism and preparing a report on that topic.

We demonstrated that a mild deficiency in dietary total amino acids induces fat accumulation in visceral and ectopic, but not subcutaneous, fat. Increases in ectopic fat accumulation and intra-abdominal fat are thought to occur in parallel [40, 41]. Feeding a lowprotein diet to piglets leads to the deposition of intramuscular and other fat $[30,31]$. In healthy humans, a high-protein diet reduces visceral fat [2], and a highprotein/low-carbohydrate diet also lowers hepatic TG levels [33]. Therefore, dietary protein content is an important determinant of body fat deposition in mammals.

\section{Conclusions}

Our present results demonstrated that a mild deficiency in dietary total amino acids or solely in dietary arginine caused a large increase in TG accumulation in the liver via different mechanisms, although both diets lacked 
arginine to the same extent. The low Arg diet caused hepatic lipid accumulation by attenuating VLDL secretion, which may be caused, at least in part, by the downregulation of ApoA4 expression. Although the concrete molecular mechanisms remain elusive, understanding how these differences come about may explain the tissue-specific responses to dietary protein malnutrition with regard to insulin-like activity and lipid metabolism.

\section{Supplementary information}

Supplementary information accompanies this paper at https://doi.org/10. 1186/s12986-020-00477-5.

Additional file 1: Figure S1 Insulin signaling-related protein levels in rats fed the 5PAA and low Arg diets. Six-week-old male Wistar rats were fed the 15PAA $(n=8)$, low Arg $(n=8)$, or 5PAA $(n=8)$ diet for $14 \mathrm{~d}$. Whole-liver lysates were analyzed by immunoprecipitation and immunoblotting with antibodies against p85 (B), S6K (C), pS6K (D), AMPK (F), and $\operatorname{pAMPK}(G)$, where anti-S6K served as the internal control; or with antibodies against pS6K/S6K (E) and pAMPK $(\mathrm{H})$, where anti-GAPDH served as the internal control. Figure S2 Average RER values during the light period (8:00-20:00) and dark period (20:00-8:00) were calculated individually.

Additional file 2: Table S1 Plasma glucose and insulin levels in rats fed the 5PAA and low Arg diets. Table $\mathbf{S 2}$ List of apolipoprotein genes.

\section{Abbreviations}

15PAA: Diet containing amino acids from casein equivalent to $15 \%$ protein; 4E-BP1: Eukaryotic initiation factor 4E-binding protein; 5PAA: Diet containing amino acids from casein equivalent to $5 \%$ protein; ANOVA: Analysis of variance; ApoA4: Apolipoprotein A-IV; ApoB: Apolipoprotein B; FFA: Free fatty acid; IR $\beta$ : Insulin receptor subunit $\beta$; IRS2: Insulin receptor substrate 2 ; MTP: Microsomal triglyceride transfer protein; NAFLD: Non-alcoholic fatty liver disease; OGTT: Oral glucose tolerance test; PCR: Polymerase chain reaction; RER: Respiration exchange rate; RT: Reverse transcription; S6K: S6 kinase; TG: Triglyceride; VLDL: Very-low-density lipoprotein

\section{Acknowledgements}

We thank Dr. Susan Hall (University of North Carolina) for critically reading the manuscript.

\section{Authors' contributions}

L.O., H.N., S.-I.T., and H.K. were responsible for the study concept and design. L.O., H.N., A.K., and Y.A. contributed to the acquisition of animal data. Y.Ta. and Y.To. performed western blotting and analyzed the data. L.O., H.N., S.-I.T., and H.K. assisted with data analysis and the interpretation of the findings. L.O. and H.N. drafted the manuscript with input from all authors. Y.Ta., Y.To., D.Y., F.H., and H.J. contributed to the interpretation of the results. H.K. provided critical revision of the manuscript with important intellectual input. All authors critically reviewed the content and approved the final version for publication.

\section{Funding}

This work was supported by the Program for the Promotion of Basic and Applied Researches for Innovations in Bio-oriented Industry (awarded to S.I.T. and H.K.). In addition, we are grateful for the matching of funds for research and development in the field of Knowledge Integration and Innovation from the Bio-oriented Technology Research Advancement Institution of the National Agriculture and Food Research Organization, Grants-inAid for Scientific Research (S) \#25221204 and Scientific Research (A) $\# 18 \mathrm{H03972}$, and funding from the Core-to-Core Program (Advanced Research Networks) from the Japan Society for the Promotion of Science.

\section{Availability of data and materials}

The datasets used and/or analyzed during the current study are available from the corresponding author upon reasonable request.

\section{Ethics approval and consent to participate}

All experiments using animals were approved by the Animal Usage Committee of the Faculty of Agriculture of the University of Tokyo, and performed in accordance with the Guidelines for Animal Experiments of The University of Tokyo (Permission No. P09-375).

\section{Consent for publication}

Not applicable.

\section{Competing interests}

The authors declare that they have no competing interests.

\section{Author details}

${ }^{1}$ Department of Agricultural Biological Chemistry, The University of Tokyo, Bunkyo-ku, Tokyo 113-8657, Japan. ²Department of Animal Sciences, The University of Tokyo, Tokyo, Japan. ${ }^{3}$ Department of Veterinary Medical Sciences, Graduate School of Agricultural and Life Sciences, The University of Tokyo, Tokyo, Japan. ${ }^{4}$ Department of Bioregulation, Institute for Advanced Medical Sciences, Nippon Medical School, Tokyo, Japan.

Received: 17 January 2020 Accepted: 22 July 2020

Published online: 03 August 2020

\section{References}

1. Clifton PM, Bastiaans K, Keogh JB. High protein diets decrease total and abdominal fat and improve CVD risk profile in overweight and obese men and women with elevated triacylglycerol. Nutr Metab Cardiovasc Dis. 2009; 19:548-54.

2. Pesta DH, Samuel VT. A high-protein diet for reducing body fat: mechanisms and possible caveats. Nutr Metab. 2014:11:53.

3. Belobrajdic DP, Mclntosh GH, Owens JA. A high-whey-protein diet reduces body weight gain and alters insulin sensitivity relative to red meat in wistar rats. J Nutr. 2004;134:1454-8.

4. Almeida JA, Santana HAP, Motta-Santos D, Nogueira ME, Silva KKS, Miotto H, et al. High-protein diet associated with Bocaiuva supplementation decreases body fat and improves glucose tolerance in resistance-trained rats. J Med Food. 2020;23:258-65.

5. Edozien JC. Experimental kwashiorkor and marasmus. Nature. 1968;220: 917-9.

6. Soliman AT, Hassan AE, Aref MK, Hintz RL, Rosenfeld RG, Rogol AD. Serum insulin-like growth factors I and || concentrations and growth hormone and insulin responses to arginine infusion in children with protein-energy malnutrition before and after nutritional rehabilitation. Pediatr Res. 1986;20: 1122-30.

7. Winje ME, Harper AE, Benton DA, Boldt RE, Elvehjem CA. Effect of dietary amino acid balance on fat deposition in the livers of rats fed low protein diets. J Nutr. 1954:54:155-66.

8. Thissen JP, Ketelslegers JM, Underwood LE. Nutritional regulation of the insulin-like growth factors. Endocr Rev. 1994;15:80-101.

9. Toyoshima Y, Tokita R, Taguchi Y, Akiyama-Akanishi N, Takenaka A, Kato H, et al. Tissue-specific effects of protein malnutrition on insulin signaling pathway and lipid accumulation in growing rats. Endocr J. 2014;61:499-512.

10. Nishi H, Yamanaka D, Kamei H, Goda Y, Kumano M, Toyoshima Y, et al. Importance of serum amino acid profile for induction of hepatic steatosis under protein malnutrition. Sci Rep. 2018;8:5461.

11. Levine LS, Wright PG, Marcus F. Failure to secrete immunoreactive insulin by rats fed a low protein diet. Acta Endocrinol. 1983;102:240-5.

12. Thams P, Capito K. L-arginine stimulation of glucose-induced insulin secretion through membrane depolarization and independent of nitric oxide. Eur J Endocrinol. 1999;140:87-93.

13. Ferreira F, Barbosa HCL, Stoppiglia LF, Delghingaro-Augusto V, Pereira EA, Boschero AC, et al. Decreased insulin secretion in islets from rats fed a low protein diet is associated with a reduced PKA alpha expression. J Nutr. 2004; 134:63-7.

14. Li C, Najafi H, Daikhin Y, Nissim IB, Collins HW, Yudkoff M, et al. Regulation of leucine-stimulated insulin secretion and glutamine metabolism in isolated rat islets. J Biol Chem. 2003;278:2853-8.

15. Takahashi S, Kajikawa M, Umezawa T, Kato H, Miura Y, Nam TJ, et al. Effect of dietary proteins on the plasma immunoreactive insulin-like growth factor-1/somatomedin C concentration in the rat. Br J Nutr. 1990;63:521-34. 
16. Toyoshima Y, Tokita R, Ohne Y, Hakuno F, Noguchi T, Minami S, et al. Dietary protein deprivation upregulates insulin signaling and inhibits gluconeogenesis in rat liver. J Mol Endocrinol. 2010;45:329-40.

17. Narita K, Nagao K, Bannai M, Ichimaru T, Nakano S, Murata T, et al. Dietary deficiency of essential amino acids rapidly induces cessation of the rat estrous cycle. PLoS One. 2011;6:1.

18. Folch J, Lees M, Stanley GHS. A simple method for the isolation and purification of Total Lipides from animal tissues. J Biol Chem. 1957;226:497509.

19. Otway S, Robinson DS. The effect of a non-ionic detergent (triton WR 1339) on the removal of triglyceride fatty acids from the blood of the rat. J Physiol. 1967;190:309-19.

20. VerHague MA, Cheng DM, Weinberg RB, Shelness GS. Apolipoprotein A-IV expression in mouse liver enhances triglyceride secretion and reduces hepatic lipid content by promoting very low density lipoprotein particle expansion. Arterioscler Thromb Vasc Biol. 2013;33:2501-8.

21. Yue JTY, Mighiu PI, Naples M, Adeli K, Lam TKT. Glycine normalizes hepatic triglyceride-rich VLDL secretion by triggering the CNS in high-fat fed rats. Circ Res. 2012;110:1345.

22. Leavens KF, Easton RM, Shulman Gl, Previs SF, Birnbaum MJ. Akt2 is required for hepatic lipid accumulation in models of insulin resistance. Cell Metab. 2009;10:405-18.

23. Wilson CG, Tran JL, Erion DM, Vera NB, Febbraio M, Weiss EJ. Hepatocytespecific disruption of CD36 attenuates fatty liver and improves insulin sensitivity in HFD-fed mice. Endocrinology. 2016;157:570-85.

24. Liao J, Sportsman R, Harris J, Stahl A. Real-time quantification of fatty acid uptake using a novel fluorescence assay. J Lipid Res. 2005;46:597-602.

25. Wang F, Kohan AB, Lo CM, Liu M, Howles P, Tso P. Apolipoprotein A-IV: a protein intimately involved in metabolism. J Lipid Res. 2015;56(8):1403.

26. Koo SH. Nonalcoholic fatty liver disease: molecular mechanisms for the hepatic steatosis. Clin Mol Hepatol. 2013;19:210-5.

27. Kubota N, Kubota T, Itoh S, Kumagai H, Kozono H, Takamoto I, et al. Dynamic functional relay between insulin receptor substrate 1 and 2 in hepatic insulin signaling during fasting and feeding. Cell Metab. 2008;8:4964.

28. Taniguchi CM, Ueki K, Kahn R. Complementary roles of IRS-1 and IRS-2 in the hepatic regulation of metabolism. J Clin Invest. 2005;115:718-27.

29. Dong X, Park S, Lin X, Copps K, Yi X, White MF. Irs1 and Irs2 signaling is essential for hepatic glucose homeostasis and systemic growth. J Clin Invest. 2006;116:101-14.

30. Wood JD, Nute GR, Richardson RI, Whittington FM, Southwood O, Plastow $\mathrm{G}$, et al. Effects of breed, diet and muscle on fat deposition and eating quality in pigs. Meat Sci. 2004;67:651-67.

31. da Costa N, McGillivray C, Bai Q, Wood JD, Evans G, Chang KC. Restriction of dietary energy and protein induces molecular changes in young porcine skeletal muscles. J Nutr. 2004;134:2191-9.

32. Katsumata M. Promotion of intramuscular fat accumulation in porcine muscle by nutritional regulation. Anim Sci J. 2011;82:17-25.

33. Martens EA, Gatta-Cherifi B, Gonnissen HK, Westerterp-Plantenga MS. The potential of a high protein-low carbohydrate diet to preserve intrahepatic triglyceride content in healthy humans. PLoS One. 2014;9:e109617.

34. Su X, Abumrad NA. Cellular fatty acid uptake: a pathway under construction. Trends Endocrinol Metab. 2009;20:72-7.

35. Borensztajn J, Rone MS, Kotlar TJ. The inhibition in vivo of lipoprotein lipase (clearing-factor lipase) activity by triton WR-1339. Biochem J. 1976;156:53943.

36. Tiwari S, Siddiqi SA. Intracellular trafficking and secretion of VLDL. Arterioscler Thromb Vasc Biol. 2012;32:1079-86.

37. Barbosa S, Fasanella G, Carreira S, Llarena M, Fox R, Barreca C, et al. An orchestrated program regulating secretory pathway genes and cargos by the transmembrane transcription factor CREB-H. Traffic. 2013;14:382-98.

38. Nagasawa M, Hara T, Kashino A, Akasaka Y, Ide T, Murakami K. Identification of a functional peroxisome proliferator-activated receptor (PPAR) response element (PPRE) in the human apolipoprotein A-IV gene. Biochem Pharmacol. 2009;78:523-30.

39. Katsumata M, Kobayashi $H$, Ashihara A, Ishida A. Effects of dietary lysine levels and lighting conditions on intramuscular fat accumulation in growing pigs. Anim Sci J. 2018;89:988-93.

40. Heilbronn L, Smith SR, Ravussin E. Failure of fat cell proliferation, mitochondrial function and fat oxidation results in ectopic fat storage, insulin resistance and type II diabetes mellitus. Int J Obes Relat Metab. 2004; 28(Suppl 4):S12-21.

41. Hocquette JF, Gondret F, Baeza E, Medale F, Jurie C, Pethick DW. Intramuscular fat content in meat-producing animals: development, genetic and nutritional control, and identification of putative markers. Animal. 2010; 4:303-19.

\section{Publisher's Note}

Springer Nature remains neutral with regard to jurisdictional claims in published maps and institutional affiliations.
Ready to submit your research? Choose BMC and benefit from:

- fast, convenient online submission

- thorough peer review by experienced researchers in your field

- rapid publication on acceptance

- support for research data, including large and complex data types

- gold Open Access which fosters wider collaboration and increased citations

- maximum visibility for your research: over $100 \mathrm{M}$ website views per year

At BMC, research is always in progress.

Learn more biomedcentral.com/submissions 